\title{
Ein Fall für zwei: ÖSG fordert Rechtsanspruch auf ärztliche Zweitmeinung
}

\begin{abstract}
Mehr Patientensicherheit, weniger medizinische Fehleinschätzungen oder unnötige Eingriffe: Im Zweifelsfall wäre eine zweite ärztliche Meinung oft ein Gewinn. Doch noch gibt es keinen gesetzlichen Anspruch auf eine Zweitmeinung in Österreich. Die Österreichische Schmerzgesellschaft (ÖSG) möchte dies gerne ändern.
\end{abstract}

Die Diagnose ist unklar. Es gibt eine Vielzahl an Therapiemöglichkeiten. Oder: Die ärztliche Einschätzung sollte zur Sicherheit noch einmal bestätigt werden. Es gibt viele gute Gründe, warum sich Patient*innen im Zweifelsfall eine zweite ärztliche Meinung wünschen. In Österreich kann bei der Österreichischen Gesundheitskasse (ÖGK) in Eigeninitiative ein Antrag auf Zweitmeinung gestellt und argumentiert werden, warum diese für notwendig erachtet wird. Anders als etwa in Deutschland gibt es aber keinen Rechtsanspruch darauf. Wird der Antrag abgelehnt, müssen Patient*innen die ärztliche Leistung aus eigener Tasche bezahlen.,,Die ÖSG möchte das ändern - wie viele andere medizinische Fachgesellschaften auch. Wenn sich Patient ${ }^{*}$ innen hinsichtlich Diagnose oder Therapieempfehlung nicht sicher sind, sollten sie das Recht auf eine Zweitmeinung haben“, sagt ÖSG-Präsidentin OÄ Dr. Waltraud Stromer anlässlich der 21. Österreichischen Schmerzwochen. In der Regel erstellen Spezialist*innen eindeutige Diagnosen, denen Patient*innen vertrauen können. .Vier Augen sehen aber mehr als zwei. Bei Unklarheiten eine zweite Meinung einzuholen, ist auch im Interesse der behandelnden Ärzt*innen und kein Zeichen von Inkompetenz oder Unsicherheit. In bestimmten Fällen empfehlen gute Ärzt*innen von sich aus eine Zweitmeinung", sagt Dr. Stromer.

Mit Zweitmeinungen lassen sich außerdem Ressourcen und Geld sparen. Eine Studie aus Deutschland zeigt, dass Wirbelsäulenoperationen zwar innerhalb von zehn Jahren um $71 \%$ zugenommen haben, jedoch sehr oft medizinisch nicht erforderlich sind. Im Zuge der Studie wurden mit einem Zweitmeinungsverfahren Operationsindikation und konservative Therapiealternativen überprüft. Dabei wurde nur bei 7,7\% der Fälle die Notwendigkeit der Operation bestätigt. Bei rund $45 \%$ der Patient*innen wurde alternativ eine besondere Versorgung im Rahmen eines ambulanten multimodalen Intensivprogramms empfohlen, bei beinahe der Hälfte der Betroffenen die Fortführung der Behandlung als ausreichend angesehen. In neun von zehn Fällen war die Absage einer kosten- und ressourcenintensiven Operation endgültig.

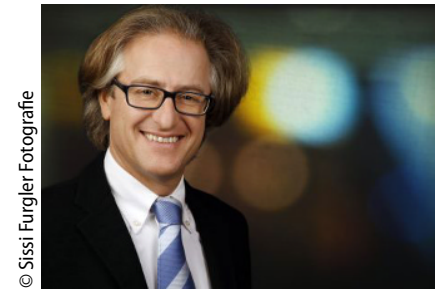

A Prim. Doz. Dr. Gerd Ivanic, Graz

\section{In vielen Fachbereichen etabliert}

In Österreich ist es in vielen medizinischen Fachbereichen bereits gang und gäbe, sich mit Kolleg*innen zu beraten, wie die beste Therapie aussehen kann. Prim. Doz. Dr. Gerd Ivanic, ÖSG-Vorstandsmitglied und Leiter des Institutes für Orthopädische und Kardiologische Rehabilitation an der Privatklinik Graz Ragnitz, verweist auf die Vorgangsweise in seinem Fachgebiet:„Als Bindeglied zwischen konservativer und operativer Orthopädie arbeite ich oft und selbstverständlich mit Zweitmeinungen, etwa bei der Behandlung von Bandscheibenvorfällen." Hier gibt es seltene Fälle, in denen unbedingt und rasch operiert werden muss, etwa wenn Bandscheibengewebe die Nerven so stark beeinträchtigt, dass die Blase oder der Darm nicht mehr funktioniert oder bestimmte Muskeln geschwächt sind. „Dann gilt es, keine Zeit zu verlieren, und das Einholen einer Zweitmeinung wäre nicht im Sinne der optimalen Behandlung", sagt der Experte. Bei anderen Bandscheibenvorfällen können Ärzt*innen das Für und Wider einer Operation mit den Patient*innen aber sorgfältig abwägen. Es gibt auch eine hohe Ziffer von notwendigen, aber nicht oder zu spät durchgeführten Operationen. Mit dem Einholen einer zweiten Meinung wird einerseits verhindert, dass ein Eingriff zu früh indiziert wird, , andererseits kann die Zweitmeinung auch zum Beispiel bei infausten konservativen Behandlungen - zur Operation führen, um ein für die Patient*innen zufriedenstellendes Ergebnis zu bringen. Das Einholen einer zweiten ärztlichen Meinung gehört zum guten medizinischen Ton, der für den Behandlungserfolg eine große Rolle spielt", resümiert Dr. Ivanic.

\section{Nachholbedarf im niedergelassenen Bereich}

Im niedergelassenen Bereich ist das Vier-Augen-Prinzip hingegen nach wie vor die Ausnahme. Nur in den wenigen Gruppenpraxen ist die zweite Meinung einer Fachkolleg*in innerhalb derselben Ordination bereits Routine. Damit das Prinzip der Zweitmeinung breiteren Eingang in die medizinische Praxis findet, schlägt ÖSG-Präsidentin Dr. Stromer vor: „Ärztekammer, Patientenanwaltschaft und Krankenversicherungsträger sollten sich zusammenfinden und gemeinsam Standards entwickeln sowie die finanzielle Abdeckung für Zweitmeinungen sicherstellen."

Bericht:Dr. Stefan Wolfinger

Quelle: Überall M, Nolte T, Kletzko H, Müller-Schwefe G: Operationen bei chronischen Rückenschmerzen nur in 7,7\% indiziert! Ergebnisse des IMC Zweitmeinungsverfahrens bei 3824 Patienten mit bereits terminierten Rückenoperationen. Abstract P11.01. Der Schmerz 2019; 1:73

Hinweis des Verlags. Der Verlag bleibt in Hinblick auf geografische Zuordnungen und Gebietsbezeichnungen in veröffentlichten Karten und Institutsadressen neutral.

Schmerz Nachr 2022 $222: 12$

https://doi.org/10.1007/s44180-022-00013-2

Online publiziert: 24 . Februar 2022

(c) The Author(s), under exclusive licence to Springer-Verlag GmbH Austria, ein Teil von Springer Nature 2022 